\title{
ESSG-BASED GLOBAL SPATIAL REFERENCE FRAME FOR DATASETS INTERRELATION
}

\author{
Jieqing $\mathrm{Yu}^{\mathrm{a}} \quad{\text { Lixin } \mathrm{Wu}^{\mathrm{a}} \mathrm{b}^{*}}$ Yongji Jia $^{\mathrm{a}}$ \\ a School of Environment Science and Spatial Informatics, China University of Mining \& Technology, Xuzhou, Jiangsu, \\ P. R. China. (yujieqing@gmail.com) \\ b Academy of Disaster Reduction and Emergency Management, Beijing Normal University, Beijing, P.R. China.
}

(awulixin@263.net)

Commission: IV-WG5

KEY WORDS: global spatial reference frame, earth system spatial grid, SDOG-R, interrelation, coordinate transformation

\begin{abstract}
:
To know well about the highly complex earth system, a large volume of, as well as a large variety of, datasets on the planet Earth are being obtained, distributed, and shared worldwide everyday. However, seldom of existing systems concentrates on the distribution and interrelation of different datasets in a common Global Spatial Reference Frame (GSRF), which holds an invisble obstacle to the data sharing and scientific collaboration. Group on Earth Obeservation (GEO) has recently established a new GSRF, named Earth System Spatial Grid (ESSG), for global datasets distribution, sharing and interrelation in its 2012-2015 WORKING PLAN.The ESSG may bridge the gap among different spatial datasets and hence overcome the obstacles. This paper is to present the implementation of the ESSG-based GSRF. A reference spheroid, a grid subdvision scheme, and a suitable encoding system are required to implement it. The radius of ESSG reference spheroid was set to the double of approximated Earth radius to make datasets from different areas of earth system science being covered. The same paramerters of positioning and orienting as Earth Centred Earth Fixed (ECEF) was adopted for the ESSG reference spheroid to make any other GSRFs being freely transformed into the ESSG-based GSRF. Spheroid degenerated octree grid with radius refiment (SDOG-R) and its encoding method were taken as the grid subdvision and encoding scheme for its good performance in many aspects. A triple $(\mathrm{C}, \mathrm{T}, \mathrm{A})$ model is introduced to represent and link different datasets based on the ESSG-based GSRF. Finally, the methods of coordinate transformation between the ESSGbased GSRF and other GSRFs were presented to make ESSG-based GSRF operable and propagable.
\end{abstract}

\section{INTRODUCTION}

Earth system is a complex system with muti-sphere structure coupled, which includes the core, the mantle, the lithosphere, the atomosphere and the magnetosphere. Each of them interacted with others strongly by the exchange of matter and energy, which causes of lots of geo-phenomenons, such as volcanic explosion, earthquake, global change, and El Niño. To make clear on mechanisms and tendency on them, scientists of different subjects need to work collaboratively to sovle the problems together.

Taking earthquake as an example, geologists, geophysicists, geographic and atmospheric scientists make a detailed investageion and monitoring on the underground geological structure, the earth sufurce and above situation on the high risk region of earthquake in their own ways and formats, and then process them into a well-accepted dataset, and pass them to seismologists. Seismologists then use these data to make further analysis to make sure whether there is an earthquake or anomaly, when will it happen, and how big it will be, and finally give their results to the goverments or release them to the public. As seen above, the most important thing behind this scientific collaboration is the exchange and sharing of the data. As known, all there datasets obtained by the scientists are highly related with spatial information, which means that a global spatial reference frame(GSRF) is needed in order to reprensent, record, share and interrelates these datasets.

GSRF is a coordinate system that references the location of objects on the Earth. It enables the spatial information describable in the forms of digital, and hence makes the spatial data exchangeable between data providers and users. Generally, GSRF can be divided into Earth-Centred Earth-Fixed (ECEF) reference system, geodetic reference system (GRS), projectionbased reference system (PRS). ECEF is a three-dimensional (3D) Cartesian coordinate system. Its origin is the center of mass of the Earth, hence the name Earth-Centered. Its axes are aligned with the International Reference Pole (IRP) and International Reference Meridian (IRM) that are fixed with respect to the surface of the Earth, hence the name Earth-Fixed. However, the Earth is an ellipsoid-like geometry shape, which makes the ECEF coordinate system very inconvinent to reference a location of an object. Based on a reference ellipsoid, GRS, such as WGS-84, NAD83, BJ-54, ITRF, allows users to use latitude, longitude, and altitude (LLA) to reference a location in the near surface more convinently. GRS can be then projected onto a plane, which turns to be PRS, such as Lambert, Gauss-Kruge and Universal Transverse Mercator (UTM). According to the way of referencing, we can divide the GSRFs into point-based reference systems, such ECEF and latitudelongitude system, and grid-based reference systems, such as latitude-longitude grid (LLG) and Discrete Global Grid (DGG) (Sahr, 2003). Anyway, grid-based reference system alway depends on point-based reference system. For example, LLG is a kind of space division in the Latitude-longitude system. For the nature of sampling in grid, and the way of most spatial data acquiring and producing, such as remote sening, more and more datasets are distributed and shared in grid-based reference system rather than point-based reference system. 
Owing to rapid development of earth observation technology, human are able to obtain a large volume of, as well as a large variety of, datasets about the planet Earth. To take full use of the datasets, many international organizations, such as World Data Center (WDC), Committee on Data for Science and Technology (CODATA), and International Oceanographic Data and Information Exchange (IODE), World Meteorological Organization (WMO), has been founded, and aslo some national, regional, and international projects, such as China data sharing infrastructure of Earth System Science project (http://www.geodata.cn), INSPIRE project (http://inspire.jrc.ec.europa.eu/), and Earth System Grid (http://www.earthsystemgrid.org/) have been set up to share scientific data nationally, regionally and globally. However, seldom of them concentrate on the linkage of all these dataset in a common GSRF, which may hold an invisble obstacle to the data sharing and scientific collaboration.

Group on Earth Observations (GEO) is a governmental and international level orgnization which intends to build a Global Earth Observation System of Systems (GEOSS) and to share and interrelate the worldwide datasets freely and globally. It has recently established a new GSRF, named Earth System Spatial Grid (ESSG), for global datasets sharing in its 2012-2015 WORKING

PLAN (http://www.earthobservations.org/docshow.php?id=129). The ESSG will bridge the gap among differnets spatial datasets, and hence overcome the obstacles of data sharing. This paper is to present the implementation of the ESSG-based GSRF, including the definition of the reference spheroid, the choice of the grid subdvision and encoding schemes, the way of interrelating different datasets, and the methods of transforming the ESSGbased GSRF into and from other GSRFs.

\section{IMPLEMENTATION OF THE ESSG-BASED GSRF}

ESSG is a global 3D grid with a reference spheroid of definite position and orientation, and an efficient coding system aiming to provide a framework for earth system datasets representation, storage, distribution, interrelation, visualization, sharing and even simulation. Three compontents are necessary to build an ESSG-based GSRF: a) a reference spheroid; b) a grid subdivision scheme; c) an efficient code system.

\subsection{A REFERENCE SPHEROID FOR ESSG}

The shape of the Earth is neither ellipsoid nor spheroid. The reason why a reference ellipsoid is chosed is mostly for the convenience of converting the surveying data on the Earth surface. However, to the view of data represention, distribution, interrelation, visualization, sharing and simulation, it make no sense to distinguish a reference ellipsoid or a reference spheroid. Since mathematical operations on an ellipsoid are more complicated than that on a spheroid, we take the spheroid as the basic reference. The average radius of the Earth is about 6370 $\mathrm{km}$. However, if such spheroid is taken as the conceptional spheroid of the ESSG, many areas, such as atmosphere and ionosphere, will be not be covered. In order to take enough coverage, a spheroid with radius of $12,800 \mathrm{~km}$, nearly double of the approximate equator radius of the Earth, which is adopted as $6,400 \mathrm{~km}$ in consideration of convenience of bi-sectional subdivision and computation, is chosen as the conceptional spheroid.

Position and orientation are required for a conceptional spheroid to be a reference spheroid. In order to be freely transformed into and from other GSRFs, parameters for the position and orientation need to be consistent with the mostly adopted GSRF (e.g., ECEF). Here, we adopted the same parameters as ECEF for the reference spheroid (Figure 1), which are lised as following:

\section{- Origin}

The origin of the reference spheroid is set to be the center of mass of the Earth.

- $\quad$ Prime meridian

The meridian of zero longitude of the spheroid is the IERS Reference Meridian which is maintained by the International Earth Rotation and Reference Systems Service (IERS).

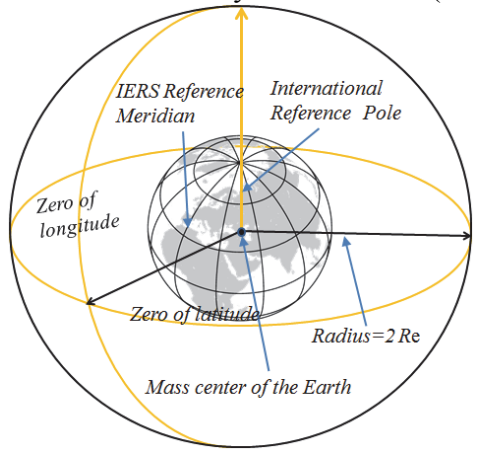

Figure 1 Reference spheroid for ESSG-based GSRF. The origin and the prime meridian set to be the center of mass of the Earth and the IERS Reference Meridian, respectively. The rotation axis is pointed to the International Reference Pole (IRP), and the radius of the reference spheroid is set to be $2 R e$ $(R e=6,400 \mathrm{~km})$.

- $\quad$ Rotation axis

The rotation axis is pointed to the International Reference Pole (IRP) as defined by IERS, which is the mean position of the Earth's spin axis between 1900.0 and 1905.0.

\subsection{GRID SUBDIVISION SCHEME FOR THE ESSG}

What kind of grid should be achieved for an ESSG-based GSRF? It is an open question. Wu (2012) has proposed several criterions to construct an ideal ESSG, which are spherical structure, geographical consistency, exhaustive coverage, hierarchical structure, approximate size, definite frame, unique coding and multiple granularities. Just as the criterions proposed by Goodchild (1992) and Kimerling (1999) to DGGs, it is hardly to find such an ideal implementation for the ESSG.

Spheroid degenerated-octree grid with refinement on radius (SDOG-R) (Yu \& Wu, 2012) satisfies most of the criterions, and can be a good realization of the ESSG. It is achieved by first subdividing the reference spheroid into Spheroid Degenerated-Octree Grid (SDOG), and then refining the radial dimension to get an SDOG-R. The two procedures are listed as following.

1) Divide the reference spheroid into eight identical octants by equatorial plane, $0^{\circ}$ meridian plane and $90^{\circ}$ meridian plane (Figure 2), and then subdivide each octant recursively in a partition method of degenerated octree ( $\mathrm{Yu} \& \mathrm{Wu}, 2009)$ to get 
a specified size SDOG (Figure 3).

2) A recursive bisection along the radial thickness direction (Yu $\& \mathrm{Wu}, 2012$ ) is taken on each grid of SDOG to get an SDOG-R when an SDOG with specific size is reached (Figure 4).
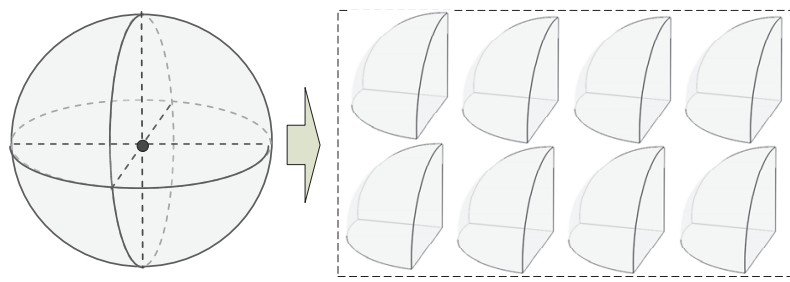

Figure 2 Dividing the reference spheroid into eight octants by equatorial plane, $0^{\circ}$ meridian plane and $90^{\circ}$ meridian plane.

\subsection{CODE SYSTEM FOR THE THE ESSG}

In order to make a good cluster for the coding system, $\mathrm{Yu}$ (2012) designed a coupled degenerated- $Z$ curving (CDZ) filling method for SDOG-R encoding. The main idea of CDZ (Figure
5) is to fill SDOG-R in a degnerated $3 \mathrm{D} \mathrm{Z}$ curve $(\mathrm{Yu}, 2009)$ on the sub-grids resulted in degenerated-octree subdivision, and to fill the sub-grids resulted in bisection with a straing line (i.e., one dimensional $\mathrm{Z}$ curve), and then join the degnerated $3 \mathrm{D} \mathrm{Z}$ curve at the order of one dimensional $Z$ curve into a continuous $\mathrm{CDZ}$ curve. The linear code of the $\mathrm{CDZ}$ curve and the octant code composed of the final code of SDOG-R.

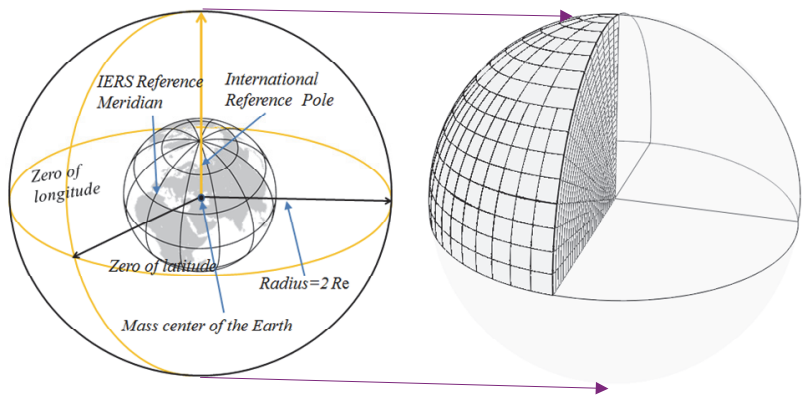

Figure 3 An octant of SDOG with a specified size.

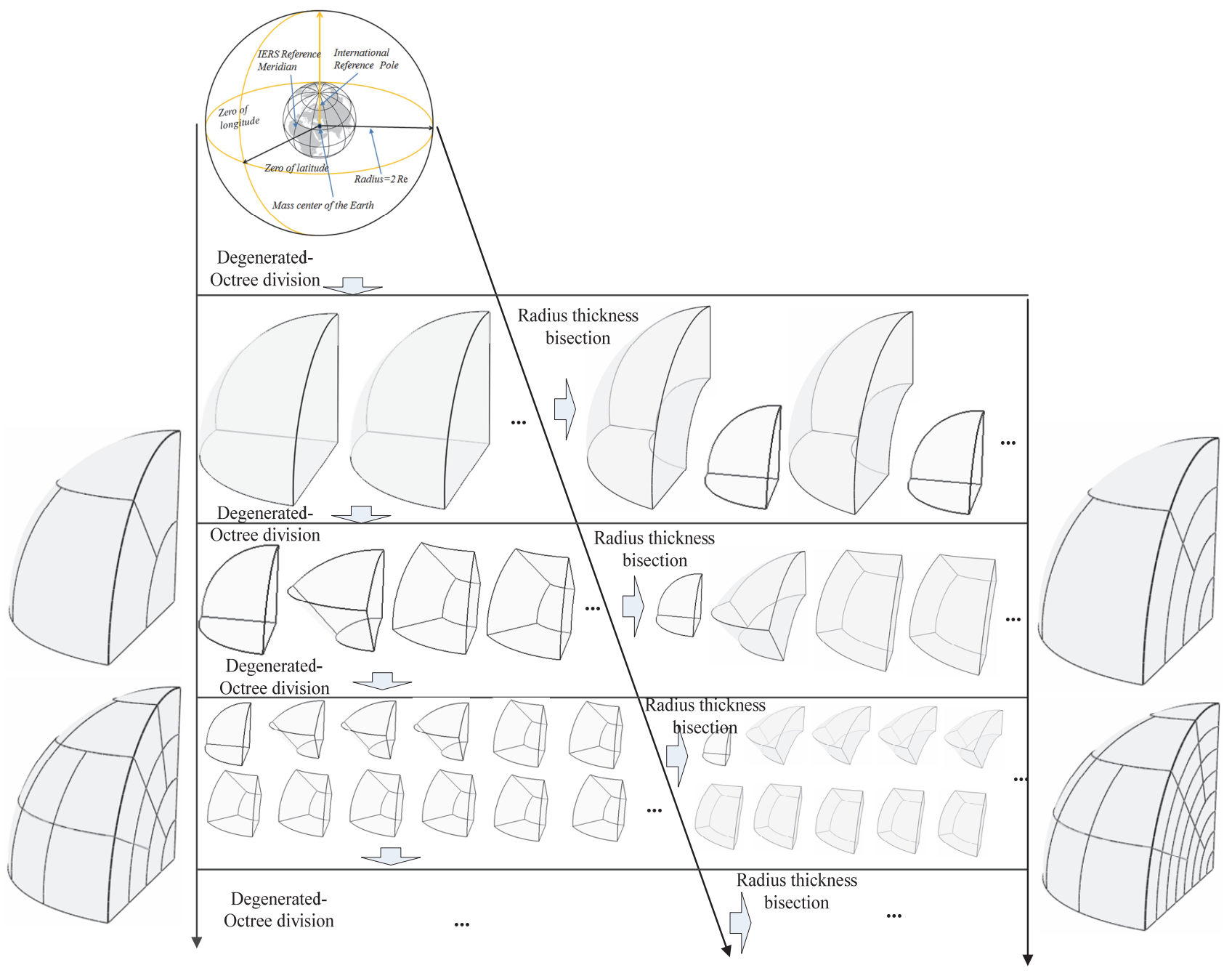

Figure 4 Hierarchical tree of SDOG-R. The SDOG-R is achieved by a recursive radial thickness bisection on an SDOG. 

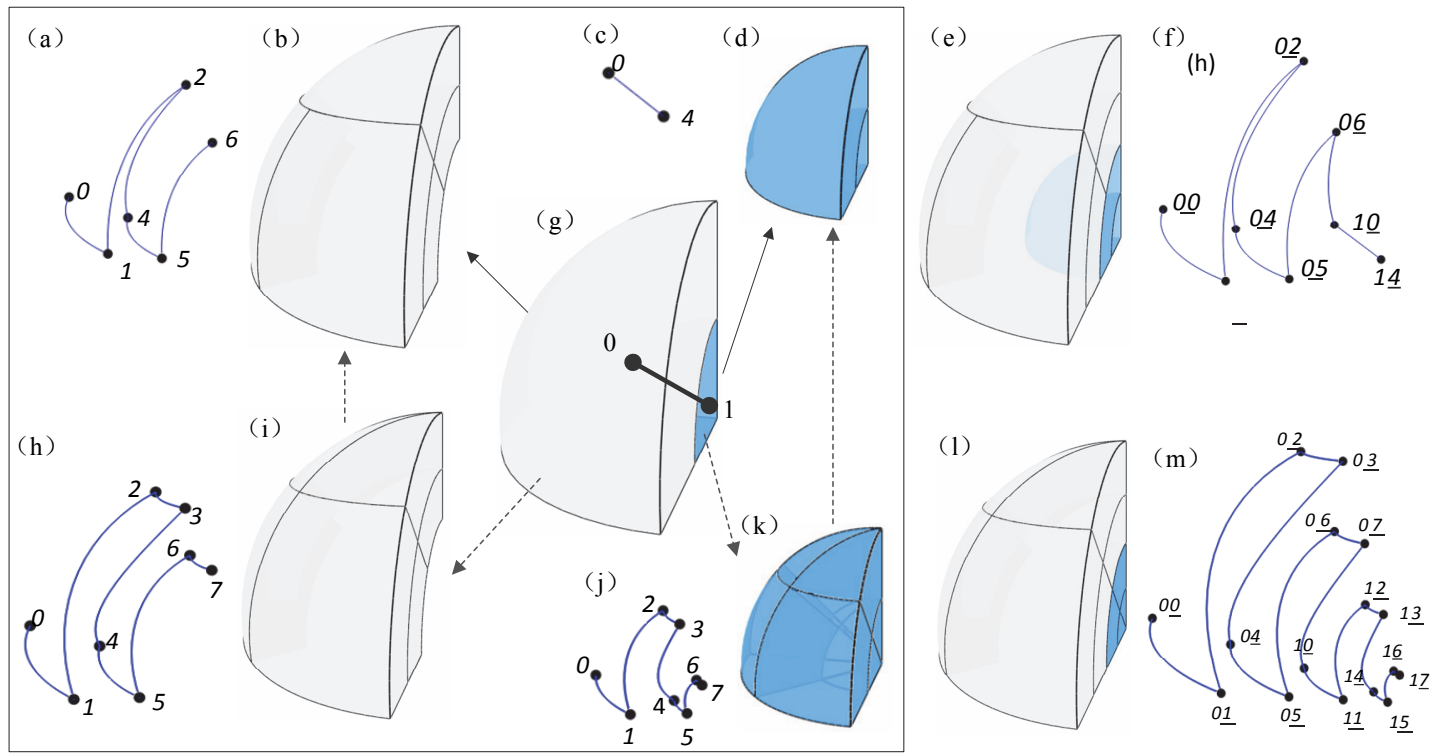

Figure 5 Methods of SDOG-R encoding. (a) The encoding scheme of the grids shown in (b); (b) the result of degenerated-octree partition on the first part of (g), which can also be achieved by local aggregation in (i); (c) the coding scheme of the grids shown in (d); (d) the result of degenerated-octree partition on the second part of (g), which can be achieved by a local aggregation in (k); (e) SDOG-R(1,1); (f) the coding scheme of SDOG-R(1,1); (g) bisection along the radial dimension on an octant or an SG; (h) the coding scheme of the grids shown in (i); (b) the result of octree partition on the first part of (g); (j) the coding scheme of the grids shown in $(\mathrm{k})$; (k) the result of octree partition on the second part of $(\mathrm{g})$; (l) the result of octree partition on $(\mathrm{g})$; (m) the coding scheme of the grids shown in (l).

\section{DATASETS INTERRELATION IN ESSG-BASED GSRF}

Each element of ESSG is a volume in the 3D space of earth system, where the dastasets are produced from. In other words, each element is a $3 \mathrm{D}$ container that holds all kind of data related to the corresponding location and granularity. Such data can either be the type, name, image, density, temperature, velocity, reflectivity, moisture, or any thing related to the space. Thus, we can employ a tuple model to link those data together with the grid element. Yu et al. $(2012 \mathrm{a} ; 2012 \mathrm{~b})$ has developed a triple model (C, T, A) (Figure 6) to link and represent the spatial, temporal and attributive information integratedly, where $\mathrm{C}$ refers to a grid code of SDOG-R, and T refers to a time stamp, and $\mathrm{A}$ refers to a collection of various attributes related to the location of the grid ' $\mathrm{C}$ ' and the time ' $\mathrm{T}$ '. With the CTA triples model, all datasets can be then represented into the same form, which is a set of large number of CTAs. Thus, all datasets can be interrelated closely at the same GSRF .

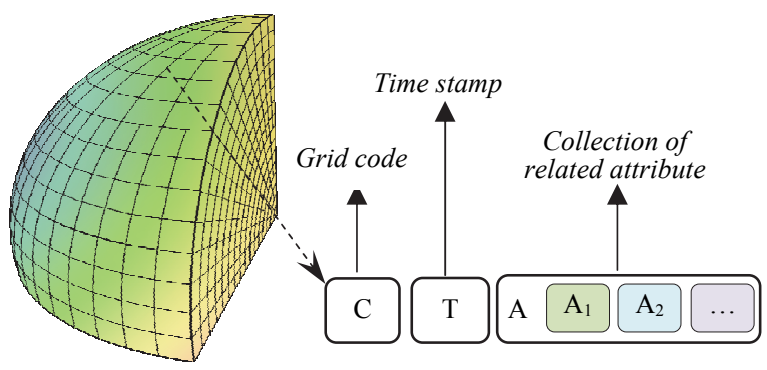

Figure 6 The CTA triple model for spatial data representation and interrelation.

\section{TRANSFORMING THE ESSG-BASED GSRF INTO AND FROM OTHER GSRFS}

Although ESSG-based GSRF is able to represent and interrelate various of datasets in a simple form, existing datasets are distributed in forms of other GSRFs. It is necessary to explore the methods of tranformation among the ESSG-based GSRF and other GSRFs. To make the transformations between ESSGbased GSRF and other GSRFs easily and clearly, a temporal reference system, called spheroid coordinate system $\operatorname{SCS})(\lambda, \phi$, $r$ ) (Figure 7a), which is highly consistent with both ECEF and ESSG, was defined in this paper. Any other GSRFs which want to be transformed into ESSG-based GSRF need to be transformed into GRS first, and then to SCS and ESSG-based GSRF (Figure 7b), vice versa. For example, when a projectionbased system like UTM wants to be converted into ESSG-based GSRF. It should be transformed into the corresponding coordinates in GRS, ECEF and SCS firstly in sequence, and then be converted into ESSG linear codes by encoding algorithm. Otherwise, the ESSG linear code should be converted into SCS coordinates firstly, and then be transformed into SCS, ECEF, and GRS coordinates in sequence.

Transformation algorithms between the projection-based system and GRS can be different accroding to different map projections. The formulations of transformation for most commonly used projections can be found in OGP (2013). More importantly, several open source libraries, such as Proj.4 (https://trac.osgeo.org/proj/), MSP GEOTRANS (http://earthinfo.nga.mil/GandG/geotrans/), $\quad$ GCTPC (http://edc.usgs.gov/pub/software/gctpc/), $\quad$ GMT (http:/gmt.soest.hawaii.edu/), were developed in the community to make the transformation between projection- 
based reference systems themselve, and between projectionbased reference system and GRSs freely. Among all those open source libraries, Proj. 4 is a widely used and stable library with a variety of map projections surpported.

(a)

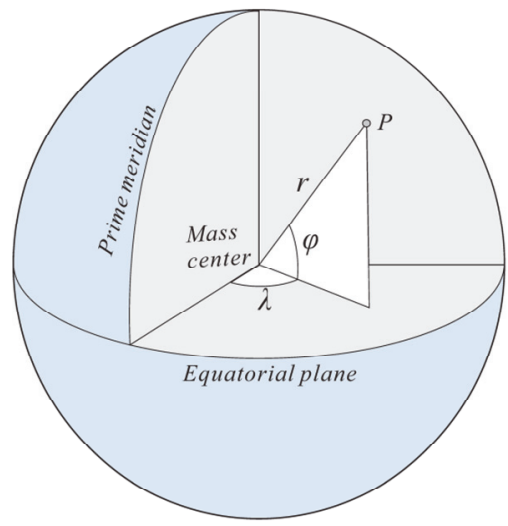

(b)

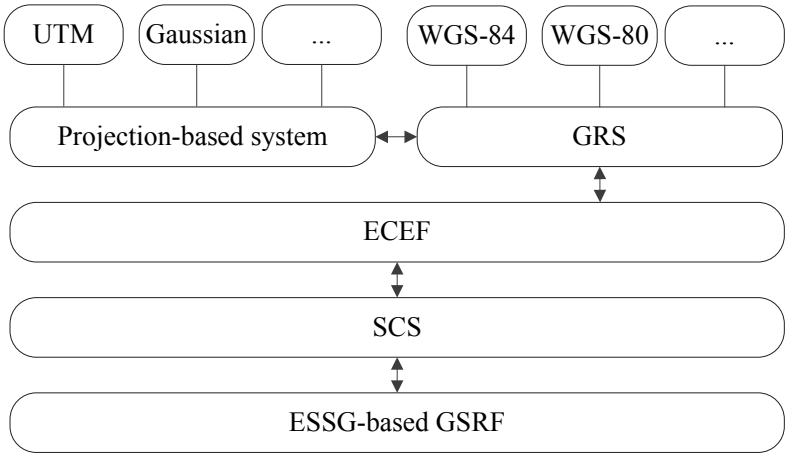

Figure 7 The definition of SCS and the transformation route between ESSG-based GSRF and other GSRFs. (a) Definition of SCS; (b) transformation route between ESSG-based GSRF and other GSRFs.

Transformation method from GRS to ECEF can be expressed as (Cai et al. 2011):

$$
\left(\begin{array}{l}
x_{e} \\
y_{e} \\
z_{e}
\end{array}\right)=\left(\begin{array}{c}
\left(N_{e}+h\right) \cos \varphi \cos \lambda \\
\left(N_{e}+h\right) \cos \varphi \sin \lambda \\
{\left[N_{e}\left(1-e^{2}\right)+h\right) \sin \varphi}
\end{array}\right),
$$

where $e$ is the first eccentricity of the reference ellipsoid, and $N_{e}$ is the prime vertical radius of curvature.

Transformation from ECEF to GRS is a little more complicated. However, we can find the method from the literatures of Nautiyal (1998), Shu and Li (2010), and Civicioglu (2012).

Transformation between ECEF and SCS is straightforward (Cai et al. 2011). Given a point in the SCS system $(\lambda, \phi, r)$, ECEF's coordinates $\left(x_{e}, y_{e}, z_{e}\right)$ can be determinated by:

$$
\begin{aligned}
& x_{e}=r \cos \varphi \cos \lambda \\
& y_{e}=r \cos \varphi \sin \lambda \\
& z_{e}=r \sin \varphi
\end{aligned}
$$

Given a point in the ECEF system $\left(x_{\mathrm{e}}, y_{\mathrm{e}}, z_{e}\right)$, SCS's coordinates $(\lambda, \phi, r)$ can be determinated by:

$$
\begin{aligned}
& \lambda=\left\{\begin{array}{c}
\arctan \frac{y_{e}}{x_{e}} \quad x_{e}>0 \\
\pi / 2 \quad x_{e}=0, y_{e}>0 \\
3 \pi / 2 \quad x_{e}=0, y_{e}<0 \\
\pi+\arctan \frac{y_{e}}{x_{e}} \quad x_{e}<0
\end{array}\right. \\
& \varphi=\left\{\begin{array}{l}
\arccos \frac{\sqrt{x_{e}^{2}+y_{e}^{2}}}{r} z_{e} \geq 0 \\
-\arccos \frac{\sqrt{x_{e}^{2}+y_{e}^{2}}}{r} z_{e}<0
\end{array}\right. \\
& r=\sqrt{x_{e}^{2}+y_{e}^{2}+z_{e}^{2}}
\end{aligned}
$$

Transformation between SCS and ESSG linear code is a process of encoding and decoding. However, ESSG linear code is a number assigned to a grid to reference a location in the Earth space rather than a vector string in ECEF and GRS. A linear code implies the location of the grid, and always refers to a region in the space while a vector string refers to a point in the space. Theoretically, it is not an one-one mapping transformation. However, any observational data refers to a certain uncertaincy. As long as the uncertaincy is given, the transformation will be one-one mapping.

Supposed that the uncentaincy of a point is $\left(\sigma_{\lambda}, \sigma_{\varphi}, \sigma_{r}\right)$, the following conditions should be met to make the transformation between SCS and ESSG linear code freely.

$$
\begin{aligned}
& \sigma_{\lambda} \geq \max \left(\delta_{\lambda}\right) \\
& \sigma_{\varphi} \geq \max \left(\delta_{\varphi}\right), \\
& \sigma_{r} \geq \max \left(\delta_{r}\right)
\end{aligned}
$$

where $\max \left(\delta_{\lambda}\right), \max \left(\delta_{\varphi}\right), \max \left(\delta_{r}\right)$ refer to the maximum size of SDOG-R on longitudinal, latitudinal and radial dimensions, respectively.

The maximum size of SDOG-R on each dimension was given by $\mathrm{Yu}$ (2012), which is shown as following:

$$
\begin{aligned}
& \max \left(\delta_{\lambda}\right)=\pi \bullet R_{s} / 2^{p+1} \\
& \max \left(\delta_{\varphi}\right)=\pi \bullet \sin \left(\pi / 2^{p+1}\right) \bullet R_{S} / 2, \\
& \max \left(\delta_{\mathrm{r}}\right)=R_{s} / 2^{p+f}
\end{aligned}
$$

where $R s$ refers to the radius of the ESSG reference spheroid, $p$ refers to the times of degenerated octree subdivision, which is named as principle subdivision level (PSL), and $f$ refers to the times of biection on SDOG, which is named as further subdivision level (FSL).

From equation 4 and 5, we can know that what kind of grid size should a point in SCS be converted into for the transformation. Once the corresponding size of grid is calculated, we can use the coding algorithms ( $\mathrm{Yu}$ 2012) to get the ESSG linear code for the point. In the opposite, we can employ a decoding algorithm ( $\mathrm{Yu}$ 2012) to convert a linear code into GRS coordinates, which are in fact a point with a $3 \mathrm{D}$ coordinate and 
an uncertaincy (grid size), and hence can be converted into the form of any other GSRF.

We've developed a tool named ESSG-Conversion for free tranformation between various GSRFs and ESSG-based GSRF. In the ESSG-conversion tool, we adopted the Proj.4 open source libarary, which enable us to freely convert any projection-based reference system into its corresponding GRS, and further convert them into ECEF, and also alows us to freely convert ECEF into any GRS and any projection-based reference system. Based on Equation 2 and 3, and the encoding and decoding algorithms given by $\mathrm{Yu}$ (2012), this tool is developed to convert ESSG-based GSRF into any other GSRF, and vice verse. The tool allows users to convert any given location in form of any GSRF into ESSG linear code, or convert any given ESSG linear code into any form of GSRF. It also allows users to convert a couple of images in form of any GSRF into ESSG CTA triples.

\section{CONCLUSION}

Many projects of earth observation have been enstablished to make an all-around, three-dimensional observation on the planet Earth since the last thirty years. Those earth observation projects produce tens of thousands of spatial datasets each year for earth science research. However, these datasets are archived or distributed in the form of different GSRFs by different holders, which vastly blocks the data sharing and collaborative research among different disciplines. Even there were many international organizations being founded to make the spatial datasets easy avaliable to the public. Seldom of them concentrate on the linkage or interrelation of these datasets in a common GSRF. To bridge the spatial gap among these spatial datasets, this paper developed an ESSG-based GSRF, which makes a variety of spatial datasets being interrelated in a common GSRF, and hence overcomes the obstacles in data sharing and collaborative reseach.

By setting the radius of reference spheroid to the double of approximated Earth radius, ESSG-based GSRF is able to cover almost all the datasets from different areas of earth system science. By adopting the same paramerters as ECEF in referecne spheroid positioning and orienting, the ESSG-based GSRF is able to be transformed into any other GSRFs freely thourgh a temporary coordinate system. By adopting SDOG-R and its encoding system as the implementation of global 3D grid system, ESSG-based GSRF is able to deal with not only two-dimensional datasets but also 3D datasets. Combing with the CTA representation model, ESSG-based GSRF is able to represent, and link all kinds of datasets in a unified way simply. And with the methods of GSRF tranformations and the ESSGConversiont tool developed in the paper, ESSG-based GSRF could be a usable and propagable GSRF for the datasets representing, distributing, interrelating, and sharing in the earth system science.

\section{Acknowledgement}

This study is joinly funded by National Natural Science Foundation of China (Grant No. 40930104), and the Fundamental Research Funds for the Central Universities (Grant No. 2013QNB10).
Cai, G., Chen, B., Lee, T., 2011. Coordinate Systems and Transformations, Unmanned Rotorcraft Systems. Springer London, pp. 23-34.

Civicioglu, P., 2012. Transforming geocentric Cartesian coordinates to geodetic coordinates by using differential search algorithm. Computers \& Geosciences, 46, pp.229-247.

Goodchild, M.F. \& Shiren, Y., 1992. A hierarchical spatial data structure for global geographic information systems. CVGIP: Graphical Models and Image Processing, 54 (1), pp. 31-44.

Kimerling, A.J., Sahr, K., White, D., et al., 1999. Comparing geometrical properties of global grids. Cartography and Geographic Information Science, 26 (4), pp., 271-288.

Nautiyal, A., 1998. Algorithm to generate geodetic coordinates from earth-fixed coordinates, Journal of Guidance, Control, and Dynamics, 11(3), pp. 281-283.

OGP, 2013. Coordinate Conversions and Transformations including Formulas, Geomatics Guidance Note, Number 7, part 12, http://www.epsg.org/guides/docs/G7-2.pdf (10 September, 2013).

Sahr, K., White, D., Kimerling, A.J., 2003. Geodesic Discrete Global Grid Systems. Cartography and Geographic Information Science, 30(2), pp. 121-134.

Shu, C., Li, F., 2010. An iterative algorithm to compute geodetic coordinates. Computers \& Geosciences, 36, pp. 11451149.

Wu, L., Yu, J., 2012. Earth System Spatial Grid and Its Application Modes. Geography and Geo-Information Science, 28(1), pp. 7-13. [In Chinese]

Yu, J. 2012. SDOG-based Earth System Spatial Grid and its application on 3D modelling, Doctoral thesis of Beijing Normal University. [In Chinese]

Yu, J. \& Wu, L., 2009. Spatial subdivision and coding of a global three-dimensional grid: Spheoid degenerated-octree grid. In: IGARSS'09, Cape Town, South africa, pp. II361-II364.

Yu, J. \& Wu, L., 2012. Adaptable spheroid degenerated-octree grid and its coding method. Geography and Geo-Information Science, 28 (1), pp. 15-18. [In Chinese]

Yu, J., Wu, L., Zi, G., et al., 2012a. SDOG-based multi-scale 3D modeling and visualization on global lithosphere. Sci China Earth Sci, 55 (6), pp. 1012-1020.

Yu, J., Wu, L., Li, Z., et al, 2012b. An SDOG-based intrinsic method for three-dimensional modelling of large-scale spatial objects. Annals of GIS, 18(4), pp. 267-278.

\section{References:}

